\title{
Noninvasive Flap Monitoring using Sidestream Dark Field Microscan: A Method for Real-Time Observation of Submilimmetric Vessels
}

\section{Ricardo A Yañez ${ }^{1 *}$, Jose R Rodriguez ${ }^{1}$, Ignacio J Cifuentes ${ }^{1}$, Bruno L Dagnino , Sebastian A Sepulveda², Arturo A Meissner ${ }^{4}$, Rodrigo A} Menchaca ${ }^{4}$ and Glenn W Hernandez ${ }^{3}$

${ }^{1}$ Section of Plastic and Reconstructive Surgery, School of Medicine, Pontificia Universidad Católica de Chile, Santiago, Chile ${ }^{2}$ Experimental Surgery and Simulation Center, School of Medicine, Pontificia Universidad Católica de Chile, Santiago, Chile ${ }^{3}$ Department of Critical Care Medicine, School of Medicine, Pontificia Universidad Católica de Chile, Santiago, Chile

${ }^{4}$ School of Medicine, Pontificia Universidad Católica de Chile

\begin{abstract}
A timely diagnosis of vascular compromise improves free flap survival. The aim of this study was to determine the feasibility of monitoring flap vascular patency through noninvasive microcirculation monitoring using SDF. An experimental study was performed in five volunteers. A radial forearm flap was simulated in both upper extremities and cutaneous microcirculation assessment devices were used (SDF and NIRS), and compared with clinical and doppler evaluation. Conditions of venous occlusion (VO) and total vascular occlusion (TVO) were mimicked by using a cuff. The results of the Microscan SDF monitoring during VO and TVO showed a reduced microcirculatory flow at 16.5 (6-30) and 6 (2-11) seconds respectively. Both NIRS and clinical evaluation were slower than SDF at identifying vascular compromise. During VO the Doppler signal does not disappear; while in TVO the signal disappeared at 1.8 (1-5) seconds. This study shows that evaluation of microcirculation with Microscan SDF is a viable alternative that may allow detection of flap venous and total vascular occlusion earlier than Doppler, NIRS and clinical evaluation.
\end{abstract}

Keywords: Microsurgery; Evaluation; Microcirculation; Monitoring

\section{Introduction}

The postoperative monitoring of free flaps following microsurgical reconstruction is as critical as the procedure itself. Flap survival rates reported in the literature are approximately $95 \%$ and above in large case series in which revision surgery was performed on patients with signs of vascular compromiso [1,2]. Evidence has shown that early detection of vascular insufficiency coupled with timely reoperation allows to rescue the flap and avoid flap failure. The reported salvage rates of flaps with anastomotic thrombosis are approximately $50 \%$ and are directly related to the time between the occlusion and surgical correction [3].

Microsurgical flaps are usually monitored by clinical evaluation, however, more objective and noninvasive methods that allow early detection of thrombotic events have been developed, including external Doppler, thermography and near infrared spectroscopy (NIRS) $[4,5]$.

Sidestream dark field (SDF) Microscan is a method for microcirculation assessment which relays on direct visualization of blood flow in capillaries. It has been used in critical care patients to evaluate resuscitation goals.

Various types of monitoring strategies have been evaluated in clinical studies. However, to date there is still no agreement on the ideal method of postoperative control for microsurgical flaps.

The purpose of this study is to determine whether monitoring microcirculation with SDF Microscan ${ }^{\circ}$ is a feasible and adequate method for detection of free flap vascular complications.

\section{Methods}

This study was conducted at the Experimental Surgery Laboratory of Pontificia Universidad Católica de Chile and was approved by the Scientific Ethics Committee - CEC MED UC (protocol no. 15-128).

Five healthy volunteers were recruited as subjects, with ages ranging from 21 to 33 years, the limits of a radial forearm flap where drawn in the distal third of both anterior forearms. A perforator vessel inside the design was identified and marked with a handheld Doppler. The baseline cutaneous microcirculation of the simulated area of the flap was assessed using the Sidestream dark field (Microscan SDF) and near infrared spectroscopy (NIRS) (Figure 1). Venous and total vascular occlusions were achieved using a cuff (Welch Allyn DS65, Skaneateles

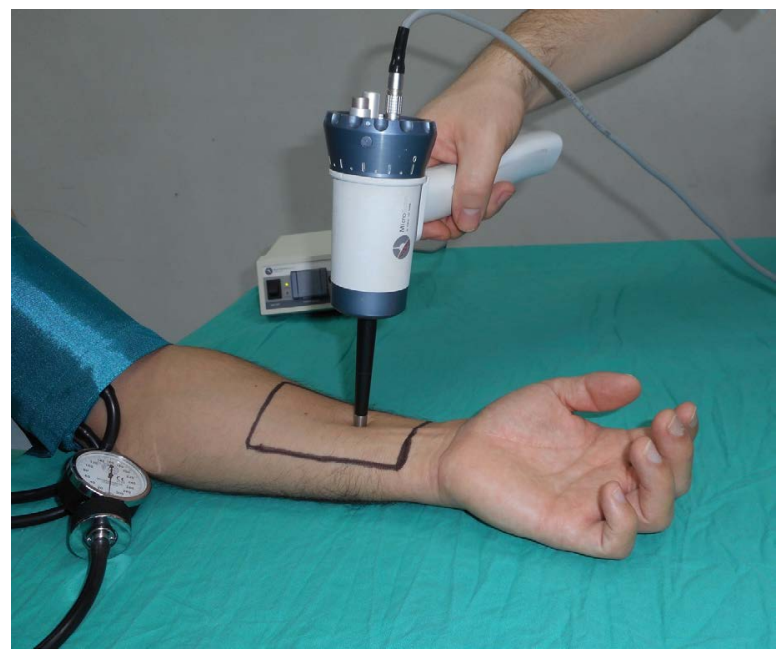

Figure 1: A radial forearm flap design was simulated in the distal third of both anterior forearms. It is possible to observe the Microscan ${ }^{\circledR}$ hand-piece.

*Corresponding author: Ricardo A Yañez, Section of Plastic and Reconstructive Surgery, School of Medicine, Pontificia Universidad Católica de Chile, Santiago, Chile, Tel: 56992209053; E-mail: ryanezm@med.puc.cl

Received: March 15, 2017; Accepted: March 22, 2017; Published:March 30 2017

Citation: Yañez RA, Rodriguez JR, Cifuentes IJ, Dagnino BL, Sepulveda SA, et al. (2017) Noninvasive Flap Monitoring using Sidestream Dark Field Microscan: A Method for Real-Time Observation of Submilimmetric Vessels. Reconstructive Surgery and Anaplastology 6: 165. doi: 10.4172/2161-1173.1000165

Copyright: @ 2017 Yañez RA, et al. This is an open-access article distributed unde the terms of the Creative Commons Attribution License, which permits unrestricted use, distribution, and reproduction in any medium, provided the original author and source are credited. 
Citation: Yañez RA, Rodriguez JR, Cifuentes IJ, Dagnino BL, Sepulveda SA, et al. (2017) Noninvasive Flap Monitoring using Sidestream Dark Field Microscan: A Method for Real-Time Observation of Submilimmetric Vessels. Reconstructive Surgery and Anaplastology 6: 165. doi: 10.4172/2161-1173.1000165

Falls, NY, USA) for 180 seconds, and changes were monitored with each method. The venous occlusion was obtained at $50 \mathrm{mmHg}$ and total vascular occlusion was obtained at $250 \mathrm{mmHg}$ as per values shown in human studies [6]. A clinical evaluation was also performed using the method standardized by Chen KT et al. (temperature, color, capillary refill). The presence or absence of flow in the perforator vessel was also monitored using a handheld sound Doppler during both venous and total vascular occlusions [5].

\section{Measurements}

a) NIRS: Oxygen saturation (StO2) is obtained by spectroscopy. We employed the Spectra StO2 Spot Check (model 300$)^{\circ}$ to collect the data. A skin sensor device was installed in the area over the perforator vessel of the simulated designed flap. The baseline register began one minute before vessel occlusion. The measurement was maintained throughout the occlusion of the vessel and $\mathrm{StO} 2$ was continuously recorded. A StO2 decrease in $50 \%$ of the baseline was considered diagnostic of impaired microcirculation $[7,8]$.

b) Microscan SDF: Polarized light videomicroscopy is a technique that allows noninvasive evaluation of microcirculation. It is a portable video-microscope (Microscan ${ }^{\circ}$, Microvision Medical, Amsterdam, Holland) that emits polarized light at $550 \mathrm{~nm}$. The light is absorbed by hemoglobin and reflected by other structures. The displayed image is obtained by contrast, and the passage of red blood cells in the microcirculation is visualized. These video images are obtained through the portable video-microscope handpiece and visualized in a monitor screen beside the subject. Then all images were recorded and analyzed. The Microvascular Flow Index (MFI) was used, which is a semiquantitative indicator that classifies the predominant flow pattern into one of 4 categories ( 0 flow absence, 1 intermittent flow, 2 slow flow and 3 normal flow). These values were previously validated for quantitative analysis of microcirculatory flow [9-11].

c) Handheld Sound Doppler was used before, during, and after occlusion of the identified perforator vessels with an pocket $8 \mathrm{MHz}$ Doppler SonoTrax Lite ${ }^{\bullet}$.

d) Temperature of the skin inside the flap design was recorded every 10 seconds during the procedure for clinical evaluation. The temperature was obtained with an infrared clinical Beurer JFT- $90^{\circ}$ Thermometer (Beurer GmbH, Ulm, Germany)

\section{Statistical analysis}

We compared the median time from vascular occlusion to a perfusion impairment diagnosis using the methods previously described from venous and total vascular occlusion.

The categorical variables were expressed as absolute numbers and percentages. The quantitative variables were reported as medians and ranges. The inferential analysis was performed using the MannWhitney/Wilcoxon test for quantitative variables and $\chi 2$ was used for categorical variables. An alpha level of $5 \%$ was used for testing. SPSS 21 data analysis software was used.

\section{Results}

Using Microscan SDF, we obtained a diminished MFI at 16.5 (6-30) seconds after vein occlusion and flow absence at 91 (29-191) seconds (Figure 2). After releasing the venous occlusion, the return of microcirculatory flow was observed at 7 (1-54) seconds (Figure 3). When a total vascular occlusion was performed, a decrease of MFI at 6 (2-11) seconds and flow absence at 13 (2-54) seconds was observed.

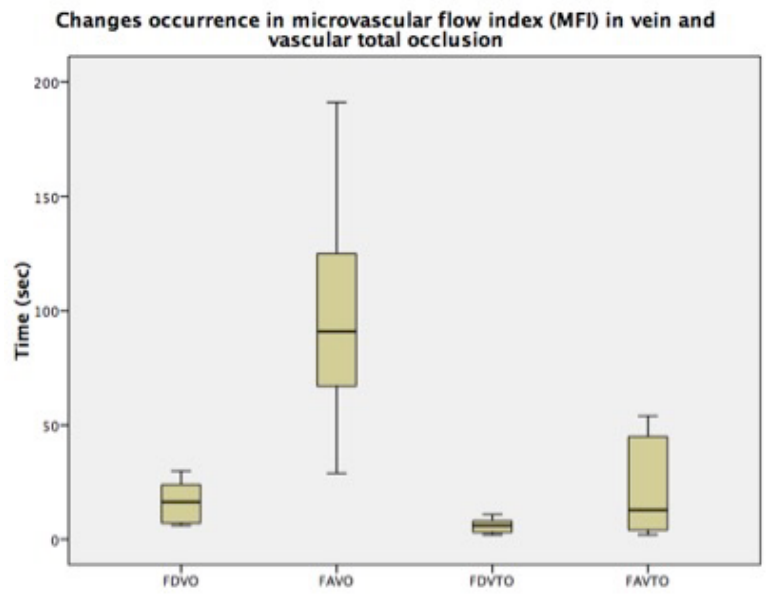

Figure 2: The microvascular flow index (MFI) changes in venous and tota vascular occlusions. The appearance of changes in the microcirculation occurred earlier in the total vascular occlusion. These differences are statistically significant. (FDVO: decreased flow in vein occlusion. FAVO: flow absence in vein occlusion. FDVTO: decreased flow in total vascular occlusion. FAVTO: flow absence in total vascular occlusion).

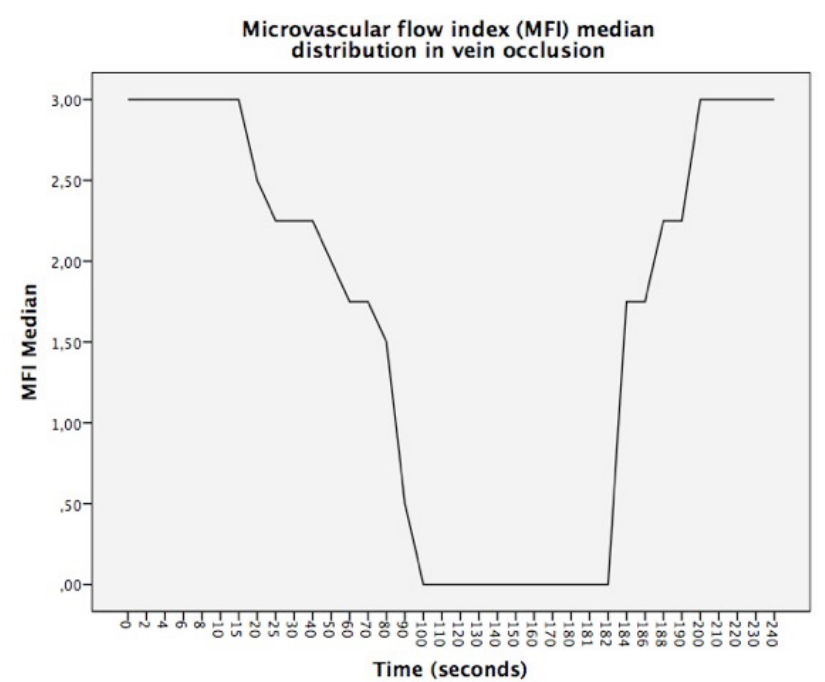

Figure 3: MFI during vein occlusion. Distribution observed in the MFI time in each subject during the test. The occlusion was performed at time 0 and then observed until flow disappeared. After 180 seconds the occlusion ends and the flow increased to normal values.

After releasing the occlusion, microcirculatory flow returned at 3.5 (1-34) seconds (Figure 4). The difference between the decrease in the microcirculatory flow for venous occlusion and total vascular occlusion was statistically significant. The venous occlusion was detected at 16.5 (6-30) seconds and total vascular occlusion was detected at $6(2-11)$ seconds $(\mathrm{p}<0.0001)$. The time between occlusion and flow absence was 91 (54-191) seconds in venous occlusion and 13 (2-54) seconds in total vascular occlusion $(p<0.009)$. The time from release of the occlusion until flow normalization was similar between venous and total vascular occlusion. The time was 7 (1-54) seconds for venous and 3.5 (1-34) seconds for total vascular occlusion $(\mathrm{p}<0.051)$. A comparison of results with SDF between each forearm was performed in the same subject and no differences were observed. After venous occlusion in the right and left forearms absence of flow occurred at 85 (29-149) seconds and 92 
Citation: Yañez RA, Rodriguez JR, Cifuentes IJ, Dagnino BL, Sepulveda SA, et al. (2017) Noninvasive Flap Monitoring using Sidestream Dark Field Microscan: A Method for Real-Time Observation of Submilimmetric Vessels. Reconstructive Surgery and Anaplastology 6: 165. doi: 10.4172/2161-1173.1000165

Page 3 of 5

(54-191) seconds respectively $(\mathrm{p}<0.26)$. The results obtained for total vascular occlusion were not significantly different at 18 (2-53) seconds for the right and 11 (4-54) seconds for the left forearm $(\mathrm{p}<0.34)$.

The following video shows the images obtained with Microscan SDF in the study: https://vimeo.com/user30321905/ microcirculationevaluationsdf.

Measurements with NIRS showed that after venous occlusion the decrease in $\mathrm{StO} 2$ percentage was $13.3 \pm 3 \%$ at 120 seconds and 19.4 $\pm 5.2 \%$ at 180 seconds. These results did not reach the criteria for perfusion abnormality (50\% decrease). In the total vascular occlusion model the $\mathrm{StO} 2$ percentage decrease was $25.6 \pm 12 \%$ at 120 seconds and $38.1 \pm 18.6 \% 180$ seconds. There was a reduction of at least $50 \%$ in $\mathrm{StO} 2$ in three out of 10 measurements performed at 158.3 (130-180) seconds (Figure 5). The difference in $\mathrm{StO} 2$ decrease between the venous

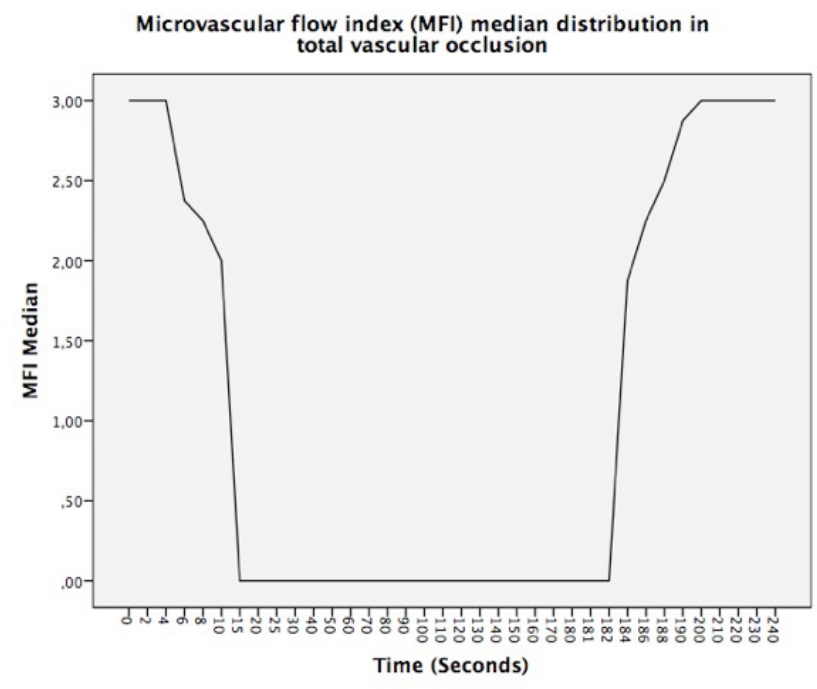

Figure 4: MFI during total vascular occlusion. Distribution observed in the MF time in each subject during the test. The occlusion was performed at time 0 and then flow was observed until it disappeared. After 180 seconds the occlusion ended and the flow returned to normal values.

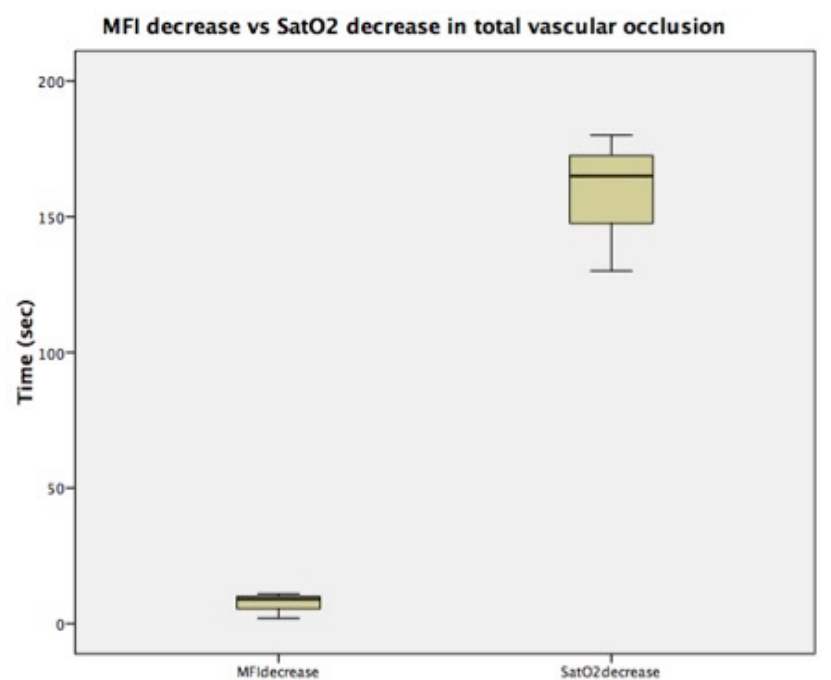

Figure 5: The comparison of MFI decrease initiation and significant SatO2 decrease in total vascular occlusions $(p<0.009)$. and total vascular occlusions at 180 seconds was statistically significant $(\mathrm{p}<0.0001)$ (Figure 6).

Evaluation of perforator vessel patency was performed using the handheld Doppler. Doppler signal did not disappear during venous occlusion and remained present throughout the procedure. When total vascular occlusion was performed, the median time until Doppler sound was lost was 1 second (1-5).

Monitoring of clinical changes during the venous occlusion phase observed congestion at $60(50-80)$ seconds, accelerated capillary refill $(<2 ")$ at $60(20-80)$ seconds, and changes in the temperature in five out of ten measurements (increase $1.2 \pm 0.3^{\circ} \mathrm{C}$ ) at 3 minutes. During total vascular occlusion congestion became apparent at 62.5 (50-150) seconds, slowed down capillary refill ( $>2$ seconds) was detected at 52.5 (40-90) seconds, pallor was reported in three out of ten measurements at $100(55-105)$ seconds, and changes in temperature were seen in four out of ten measurements (decrease $0.8 \pm 0.4^{\circ} \mathrm{C}$ ).

\section{Discussion}

The reported free flap loss in microsurgery is currently less than $5 \%$, going as low as $2 \%$ in some series [12]. Revision surgery has shown salvage rates of potential failures of up to $80 \%$ of the reoperations $[10,11]$. The evidence indicates that early detection and exploration of the flap vascular compromise increases survival rates significantly [13-16]

Several methods of postoperative flap monitoring are available today. However, there is no established standard monitoring method [17]. Early detection and treatment of vascular failure and circulatory compromise of the flap is imperative because ischemia leads to irreversible metabolic changes as time elapsed since its appearance increases $[18,19]$.

This study evaluated the feasibility of flap monitoring through noninvasive capillary microcirculation assessment with SDF which allows direct visualization of blood flow, [20] a method already widely used in shock patients in intensive care units and which requires minimal training for adequate implementation by health personnel. Microcirculation assessment has been used mainly for microsurgical monitoring in animal studies. There is only one case report of

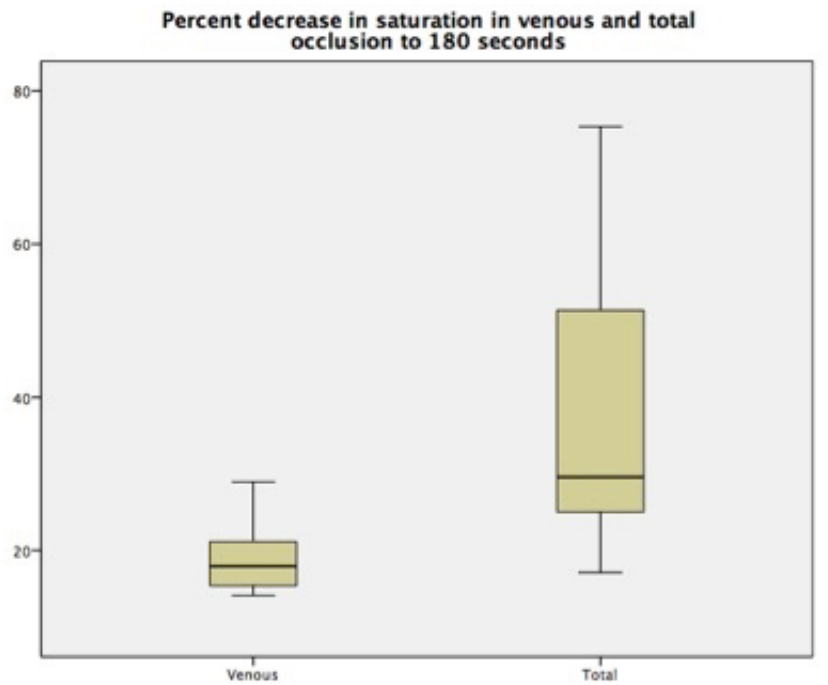

Figure 6: StO2 decrease (percentage) in venous and total vascular occlusion at 180 seconds. $(p<0.0001), 135 \times 116 \mathrm{~mm}(72 \times 72 \mathrm{DPI})$. 
Citation: Yañez RA, Rodriguez JR, Cifuentes IJ, Dagnino BL, Sepulveda SA, et al. (2017) Noninvasive Flap Monitoring using Sidestream Dark Field Microscan: A Method for Real-Time Observation of Submilimmetric Vessels. Reconstructive Surgery and Anaplastology 6: 165. doi: 10.4172/2161-1173.1000165

Page 4 of 5

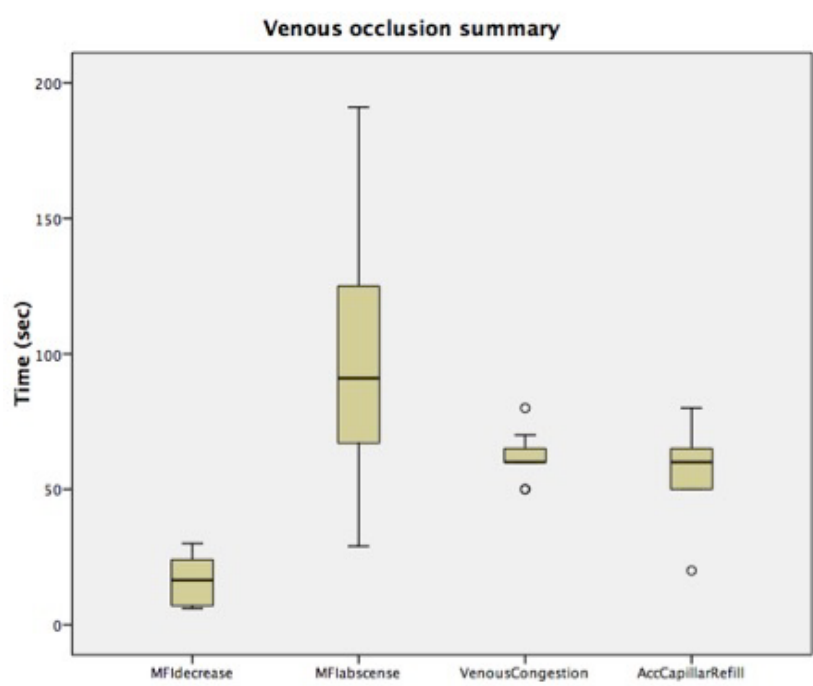

Figure 7: Summary of results obtained in venous occlusions. The disturbance of microcirculation (decreased MFI) occurred prior to the onset of congestion and accelerated capillary refill.

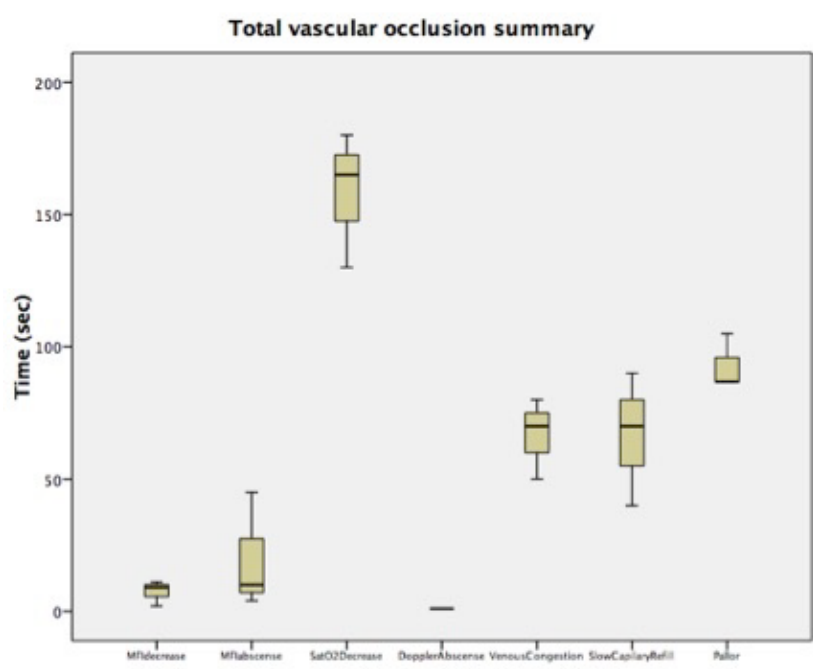

Figure 8: Summary of results obtained in total vascular occlusion. The reduction and absence of MFI anticipated clinical changes (Sat O2, congestion, slow capillary refill, pallor) except for the disappearance of the Doppler signal.

microcirculation evaluation in a DIEP flap in a human patient, that only described the method $[21,22]$.

The ability of SDF to detect impaired perfusion was tested and compared with other existing methods used for evaluation of free flap vascular status, including clinical monitoring, Doppler, and NIRS.

SDF monitoring allowed earlier diagnosis of impaired microcirculation in both venous and total vascular occlusions when compared with NIRS. There were several cases in which NIRS failed to show significant changes. The blood flow impairment detection by SDF occurred earlier than Doppler in venous occlusion. The onset of congestion and accelerated capillary refill in the flap as clinical changes were also detected later than impaired microcirculation identified by SDF (Figure 7). During total vascular occlusion loss of Doppler signal was observed earlier than absence of microcirculation flow obtained with SDF. However, the changes in microcirculation were observed before any clinical change (capillary refill, pallor) (Figure 8). Reduced venous flow is the earliest event in the vast majority of flap failures in microsurgery. Thus, SDF is potentially a useful method for early detection of flaps at risk of failure.

In a recent systematic review, it was noted that only five monitoring techniques showed evidence of increased rates of microsurgical flap rescue compared to other clinical methods (implantable Doppler, NIRS, Laser Doppler Flowmetry, quantitative fluorescence and evaluation through photographs sent by smartphones); however, none of these methods has become widely used in clinical practice [23].

Future studies are needed to adapt the method to clinical practice and evaluate results after monitoring real microsurgical flaps with SDF. These studies will determine the actual impact on clinical decisionmaking and flap survival.

If the Microscan SDF is validated for microsurgical monitoring it would be an attractive option for specific cases in daily practice, when the experience of the surgeon and the clinical assessment are unable to reliably determine the status of a microsurgical flap. Such cases might include intraoperative evaluations in very long operations, in which flap local conditions do not permit the routine clinical evaluation in a reliable manner; or when a flap which has already suffered vascular compromise prior to a second intervention proves challenging for an adequate clinical evaluation.

\section{Conclusion}

Venous and total vascular occlusion were successfully identified using Microscan SDF in an experimental simulated flap model in humans.

In the venous occlusion model, the impaired circulation was detected earlier by Microscan SDF than other methods, namely clinical assessment, Doppler and NIRS.

Microscan SDF is a feasible method to assess the perfusion status in cutaneous flaps and it should be considered as a valid alternative to other monitoring tools.

\section{References}

1. Lu JC, Zelken J, Hsu CC, Chang NJ, Lin CH, et al. (2015) Algorithmic approach to anterolateral thigh flaps lacking suitable perforators in lower extremity reconstruction. Plast Reconstr Surg 135: 1476-1485.

2. Chen KT, Mardini S, Chuang DC (2007) Timing of presentation of the first signs of vascular compromise dictates the salvage outcome of free flap transfers. Plast Reconstr Surg 120: 187-195.

3. Bui DT, Cordeiro PG, Hu QY, Disa JJ, Pusic A, et al. (2007) Free flap reexploration: Indications, treatment, and outcomes in 1193 free flaps. Plast Reconstr Surg 119: 2092-2100

4. Frost MW, Niumsawatt V, Rozen WM, Eschen GE, Damsgaard TE, et al (2015) Direct comparison of postoperative monitoring of free flaps with microdialysis, implantable cook-swartz Doppler probe, and clinical monitoring in 20 consecutive patients. Microsurgery 35: 262-271.

5. Chen Y, Shen Z, Shao Z, Yu P, Wu J, et al. (2016) Free flap monitoring using near-infrared spectroscopy: A systemic review. Ann Plast Surg 76: 590-597.

6. Crenshaw AG, Elcadi GH, Hellstrom F, Mathiassen SE (2012) Reliability of near-infrared spectroscopy for measuring forearm and shoulder oxygenation in healthy males and females. Eur J Appl Physiol 112: 2703-2715.

7. Fox PM, Zeidler K, Carey J, Lee GK (2013) White light spectroscopy for free flap monitoring. Microsurgery 33: 198-202.

8. Repez A, Oroszy D, Arnez ZM (2008) Continuous postoperative monitoring of cutaneous free flaps using near infrared spectroscopy. J Plast Reconstr Aesthet Surg 61: 71-77. 
Citation: Yañez RA, Rodriguez JR, Cifuentes IJ, Dagnino BL, Sepulveda SA, et al. (2017) Noninvasive Flap Monitoring using Sidestream Dark Field Microscan: A Method for Real-Time Observation of Submilimmetric Vessels. Reconstructive Surgery and Anaplastology 6: 165. doi: 10.4172/2161-1173.1000165

Page 5 of 5

9. Backer DD, Hollenberg S, Boerma C, Goedhart P, Büchele G, et al. (2007) How to evaluate the microcirculation: Report of a round table conference. Crit Care 11: R101.

10. Boerma EC, Mathura KR, van der Voort PH, Spronk PE, Ince C (2005) Quantifying bedside-derived imaging of microcirculatory abnormalities in septic patients: a prospective validation study. Crit Care 9: R601-R606.

11. Ruiz BC, Bruhn CA, Hernández PG, Andresen HM (2008) Evaluation of sublingual microcirculation in septic shock. Report of one patient treated with high volume hemofiltration. Rev Med Chil 136: 1175-1178.

12. Smit JM, Acosta R, Zeebregts CJ, Liss AG, Anniko M, et al. (2007) Early reintervention of compromised free flaps improves success rate. Microsurgery 27: 612-616

13. Al-Dam A, Zrnc TA, Hanken H, Riecke B, Eichhorn W, et al. (2014) Outcome of microvascular free flaps in a high-volume training centre. J Craniomaxillofac Surg 42: 1178-1183.

14. Disa JJ, Cordeiro PG, Hidalgo DA (1999) Efficacy of conventional monitoring techniques in free tissue transfer: An 11-year experience in 750 consecutive cases. Plast Reconstr Surg 104: 97-101.

15. Yang Q, Ren ZH, Chickooree D, Wu HJ, Tan HY, et al. (2014) The effect of early detection of anterolateral thigh free flap crisis on the salvage success rate, based on 10 years of experience and 1072 flaps. Int J Oral Maxillofac Surg 43: 1059-1063.

16. Chang El, Carlsen BT, Festekjian JH, Da Lio AL, Crisera CA (2013) Salvage rates of compromised free flap breast reconstruction after recurrent thrombosis. Ann Plast Surg 71: 68-71.

17. Bellamy JL, Mundinger GS, Flores JM, Wimmers EG, Yalanis GC, et al. (2015) Do adjunctive flap-monitoring technologies impact clinical decision making? An analysis of microsurgeon preferences and behavior by body region. Plast Reconstr Surg 135: 883-892.

18. Wolff KD, Stiller D (1993) Ischemia tolerance of free-muscle flaps: an NMRspectroscopic study in the rat. Plast Reconstr Surg 91: 485-491.

19. Smit JM, Zeebregts CJ, Acosta R (2008) Timing of presentation of the first signs of vascular compromise dictates the salvage outcome of free flap transfers. Plast Reconstr Surg 122: 991-992.

20. Goedhart PT, Khalilzada M, Bezemer R, Merza J, Ince C (2007) Sidestream Dark Field (SDF) imaging: A novel stroboscopic LED ring-based imaging modality for clinical assessment of the microcirculation. Opt Express 15 15101-15114.

21. Milstein DM, Lindeboom JA, Ince C (2010) Intravital sidestream dark-field (SDF) imaging is used in a rabbit model for continuous noninvasive monitoring and quantification of mucosal capillary regeneration during wound healing in the oral cavity: A pilot study. Arch Oral Biol 55: 343-349.

22. Hardwicke JT, Bella H, Waters RA (2014) Sidestream dark field imaging as an adjunct to free flap monitoring. J Plast Reconstr Aesthet Surg 67: 875-877.

23. Chae MP, Rozen WM, Whitaker IS, Chubb D, Grinsell D, et al. (2015) Current evidence for postoperative monitoring of microvascular free flaps: a systematic review. Ann Plast Surg 74: 621-632. 